\title{
Introduction to the germ line
}

\author{
E. Jane Albert Hubbard ${ }^{\S}$, Department of Biology, New York University, \\ New York, NY 10003 USA \\ David Greenstein, Department of Cell \& Developmental Biology, \\ Vanderbilt University School of Medicine, Nashville, TN 37232-2175 USA
}

\section{Table of Contents}

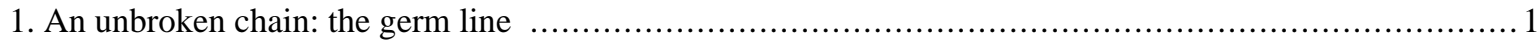

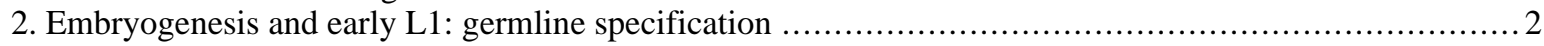

3. L1-L3: germline proliferation, survival and differentiation ............................................... 2

4. Late larval and adult stages: germline proliferation and gametogenesis .................................. 3

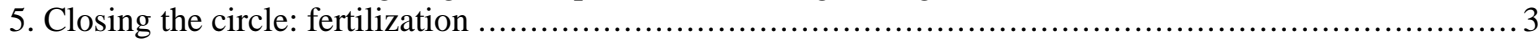

\section{An unbroken chain: the germ line}

In sexually reproducing organisms, cells of the germ line form gametes and establish a physical link, an unbroken chain, from generation to generation. C. elegans germline development can be conceptually divided into three phases: specification, growth, and maintenance. Early in embryogenesis, germ cells are specified as distinct from somatic cells. During postembryonic larval stages, the germ line proliferates and undergoes meiotic development. During the remainder of reproductive life the mature germ line continues proliferation and meiotic development, and produces gametes. Chapters in the Germ line section focus on specific molecular/developmental aspects of these events. Here, we briefly introduce these chapters in the context of the temporal progression of germline development in hermaphrodites (Figure 1).

*Edited by Judith Kimble and Susan Strome. Last revised April 09, 2005. Published September 1, 2005. This chapter should be cited as: Hubbard, E.J.A., and Greenstein, D. Introduction to the germ line (September 1, 2005), WormBook, ed. The C. elegans Research Community, WormBook, doi/10.1895/wormbook.1.18.1, http://www.wormbook.org.

Copyright: (C) 2005 E. Jane Albert Hubbard and David Greenstein. This is an open-access article distributed under the terms of the Creative Commons Attribution License, which permits unrestricted use, distribution, and reproduction in any medium, provided the original author and source are credited.

${ }^{\S}$ To whom correspondence should be addressed. E-mail: jane.hubbard@nyu.edu 
(A)

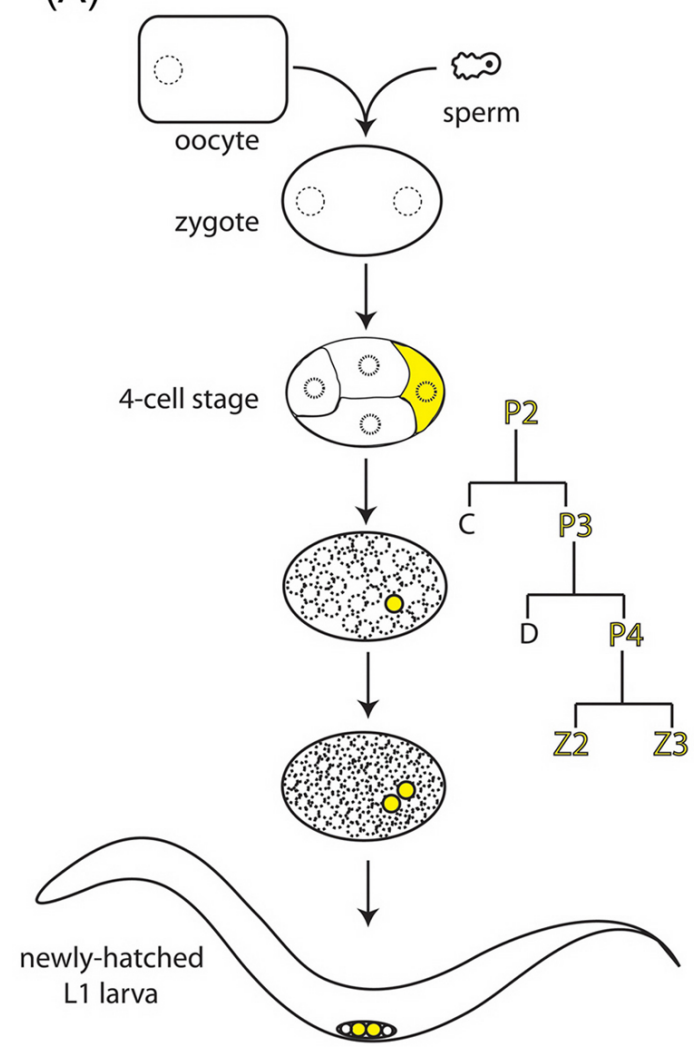

(B)

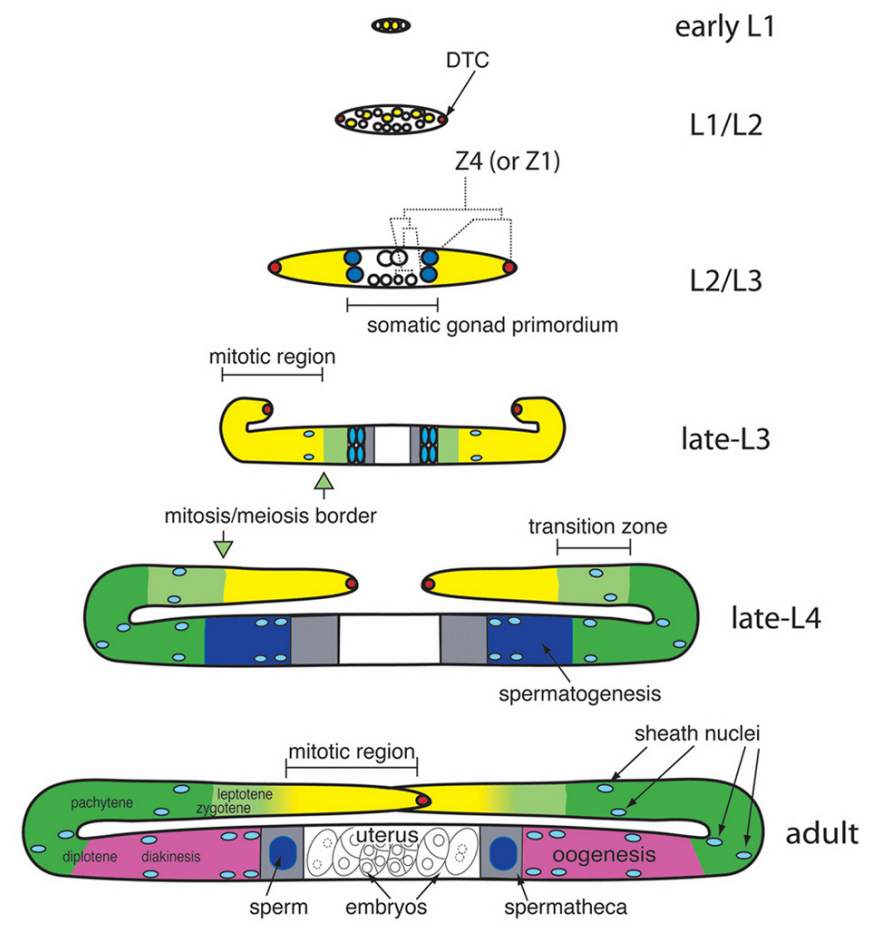

Figure 1. Cartoon representation of gonadogenesis. (A) Cartoon representation of fertilization and the embryonic germ line. Oocyte and sperm meet and fertilization initiates embryonic development. Germline lineages are depicted in yellow. (B) Cartoon representation of post-embryonic hermaphrodite gonad development. Comparative size of gonads at different stages is not to scale. Germline color scheme: yellow mitotic region, light green transition (early prophase of meiosis I), dark green pachytene, dark blue spermatogenesis, and pink oogenesis. The mitosis/meiosis border is indicated in the late-L3 and late-L4 by light green arrows. In the adult, the mitosis/meiosis border is not sharp (mitotic and meiotic nuclei are interspersed at the border) as indicated here by a yellow/green color gradient. Somatic gonad color scheme: red DTC, blue sheath/spermatheca precursor cells, light blue sheath nuclei, grey spermatheca, and white uterus. Germline nuclei and their surrounding cytoplasm are often referred to as "germ cells", though they are open to a core of shared cytoplasm (rachis) during much of their development.

\section{Embryogenesis and early L1: germline specification}

During early embryogenesis, germline potential is segregated to the P blastomeres, culminating in P4, the germline founder cell that gives rise to all of the germ cells and does not contribute to the soma. Specialized ribonucleoprotein particles, called $\mathrm{P}$ granules, are maternally provided and segregated to the $\mathrm{P}$ blastomeres. Though $\mathrm{P}$ granules are not sufficient for specifying the germ cell fate, germline development is severely impaired in the absence of their constituent proteins (see Specification of the germ line).

The PIE-1 protein plays a critical role in specifying germline fate. PIE-1 promotes germline fate in part by repressing transcriptional elongation. PIE-1 also interacts directly with MEP-1, a component of the nucleosome remodeling and deacetylase (NuRD) complex that is required in somatic cells to avoid acquisition of germline fate. The role of chromatin remodeling in the germ line has been further established by characterization of maternal-effect sterile (mes) genes and modifications of specific histone residues (see Specification of the germ line; Germline chromatin).

\section{L1-L3: germline proliferation, survival and differentiation}

At hatching, the gonad comprises the primordial germ cells (Z2 and Z3) flanked by the somatic gonad precursors (Z1 and Z4) and their surrounding basement membrane. These four cells remain mitotically quiescent until the mid-L1. nanos-related genes have been implicated in the localization of Z2 and Z3 to the gonad, maintenance of quiescence, and germline survival. $\mathrm{Z} 2$ and $\mathrm{Z3}$ also require nutritional and cell-cell signals to proliferate. Changes in chromatin modification that precede proliferation occur in response to nutritionally favorable 
conditions. Maternal mes genes are required for subsequent larval germline survival (Germline chromatin; Germline proliferation and its control).

The somatic gonad develops in concert with the germ line. Z1 and Z4 proliferate to 12 cells by the end of the L1: two distal tip cells (DTCs) that are critical for proliferation of the germ line, and 10 proximal cells that undergo a rearrangement in the late L2 to form the hermaphrodite somatic gonad primordium, which is distinct from the male somatic gonadal primordium. During formation of the somatic gonad, the germ cells are relegated to anterior and posterior populations. By the early L3, the anatomical stage is set for rapid extension of the gonadal arms, matched by robust proliferation of the germ line in response to signals from the DTCs and the developing sheath. Germline sex determination and meiotic prophase also begin in this stage (see Sex-determination in the germ line; Germline proliferation and its control).

\section{Late larval and adult stages: germline proliferation and gametogenesis}

An approximately four-fold amplification in total germ cell numbers occurs during the L4 and young adult stages. Germ cell proliferation occurs in the distal mitotic zone, a region that has been likened to a niche. GLP-1 signaling in the germ line negatively regulates meiotic entry in response to a signal from the DTC throughout larval and adult stages. The regulation of meiotic entry is complex, but involves GLD-1, GLD-2, FBF-1/2 and NOS-3, all of which are involved in post-transcriptional gene regulation (see Germline proliferation and its control; RNA regulation in the germ line). Germline proliferation, meiotic entry, and germline sex determination go hand in hand and several key genes function in multiple processes (see Sex-determination in the germ line; Germline proliferation and its control).

Once germ cells have entered the meiotic pathway, germ cell chromosomes undergo homologous recombination and synaptonemal complex (SC) formation. Chromosome pairing and alteration of nuclear organization begin in the transition zone (Figure 1) and meiotic recombination is largely completed by pachytene. As cells exit pachytene, asymmetric disassembly of the SC patterns the meiotic chromosomes for bipolar orientation on the meiotic spindle and the stepwise loss of sister chromatid cohesion during successive meiotic divisions (see Meiosis). Progression through pachytene to diplotene requires the mitogen-activated protein kinase signaling pathway in the germ line (see Control of oocyte meiotic maturation and fertilization). As meiotic chromosomes progress from diplotene to diakinesis, they become highly condensed, forming the six discrete oocyte bivalents.

Germline sex-specific differences in histone modifications occur as germ cells traverse meiotic prophase. Differences in histone modifications are most dramatic between autosomes and the X chromosome. Genome-wide transcription studies, genetics, and studies on chromatin modification have converged to suggest links between germline function, transcriptional control, and chromatin structure. Germline-enriched genes are under-represented on the $\mathrm{X}$ chromosome. Consistent with this observation, the MES-2/-3/-6 protein complex acts as a histone methyltranferase that preferentially methylates the $\mathrm{X}$ chromosome, thereby likely conferring a repressive chromatin structure (see Germline genomics; Germline chromatin).

Gametogenesis spans the late L4 and adult stages: spermatogenesis occurs during the L4 stage and oogenesis during the adult stage. As in other species, spermatogenesis produces a motile gamete. However, C. elegans spermatozoa move by a unique crawling mechanism, utilizing the major sperm protein as the chief cytoskeletal element instead of actin. Genes involved in spermatogenesis-specific controls on translation, meiosis, morphogenesis, cytoskeletal rearrangements, and signal transduction have been identified (see Spermatogenesis). Oogenesis entails a tremendous increase in cytoplasmic volume and ultimately produces a maternally provisioned cell that can support early embryogenesis. Apoptosis is a common feature of oogenesis in diverse metazoan phyla. During oogenesis in C. elegans, many female germ cells undergo the apoptotic program in the loop region of the gonad (Figure 1) and are engulfed by the gonadal sheath cells (see Control of oocyte meiotic maturation and fertilization; Germline survival and cell death). The utility of culling the oogenic germ line in $C$. elegans by apoptosis is unclear, but it may facilitate the rapid loading of the remaining oocytes with maternal factors to support embryogenesis.

\section{Closing the circle: fertilization}

The oocytes of most animal species arrest during meiotic prophase, and complete meiosis in response to intercellular signaling in a process called meiotic maturation. Oocyte meiotic maturation is defined by the transition between diakinesis and metaphase of meiosis I and is accompanied by nuclear envelope breakdown, rearrangement 
of the cortical cytoskeleton, and meiotic spindle assembly. Thus, the meiotic maturation process is an essential part of meiosis and prepares the oocyte for fertilization (see Control of oocyte meiotic maturation and fertilization).

In C. elegans, the processes of meiotic maturation, ovulation, and fertilization are temporally coupled: sperm utilize the major sperm protein as a hormone to trigger oocyte meiotic maturation, and in turn, the maturing oocyte signals its own ovulation thereby facilitating fertilization. When the non-motile spermatids enter the spermatheca during the first ovulations, they undergo spermiogenesis to form motile fertilization-competent spermatozoa. Several sperm-specific integral membrane proteins have been identified that are required in sperm for efficient fertilization (see Spermatogenesis; Control of oocyte meiotic maturation and fertilization). These final events of fertilization bring the germ line full circle.

All WormBook content, except where otherwise noted, is licensed under a Creative 